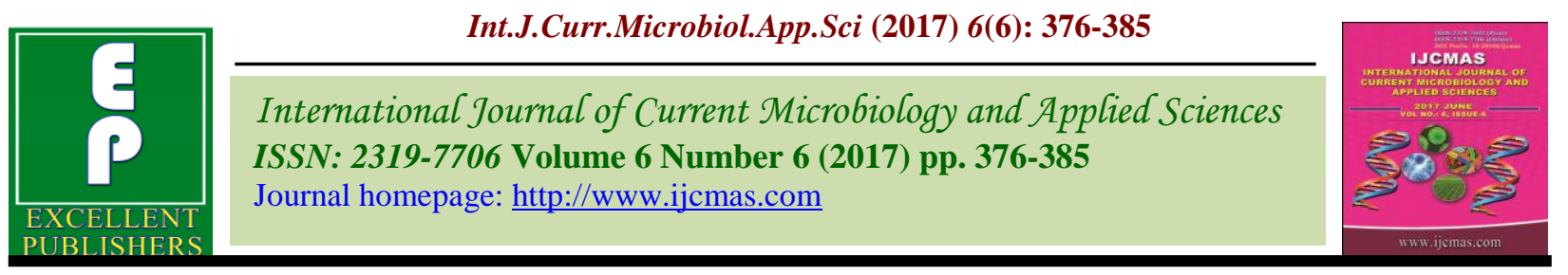

Original Research Article

https://doi.org/10.20546/ijcmas.2017.606.044

\title{
Growth and Yield of Machine Transplanted Rice (Oryza sativa L.) as Influenced by Age and Number of Seedlings
}

\author{
R.B. Negalur ${ }^{1 *}$ and A.S.Halepyati ${ }^{2}$ \\ ${ }^{1}$ AICRP on weed management, MARS, UAS Raichur, Karnataka, India \\ ${ }^{2}$ College of Agriculture, UAS, Raichur, India \\ *Corresponding author
}

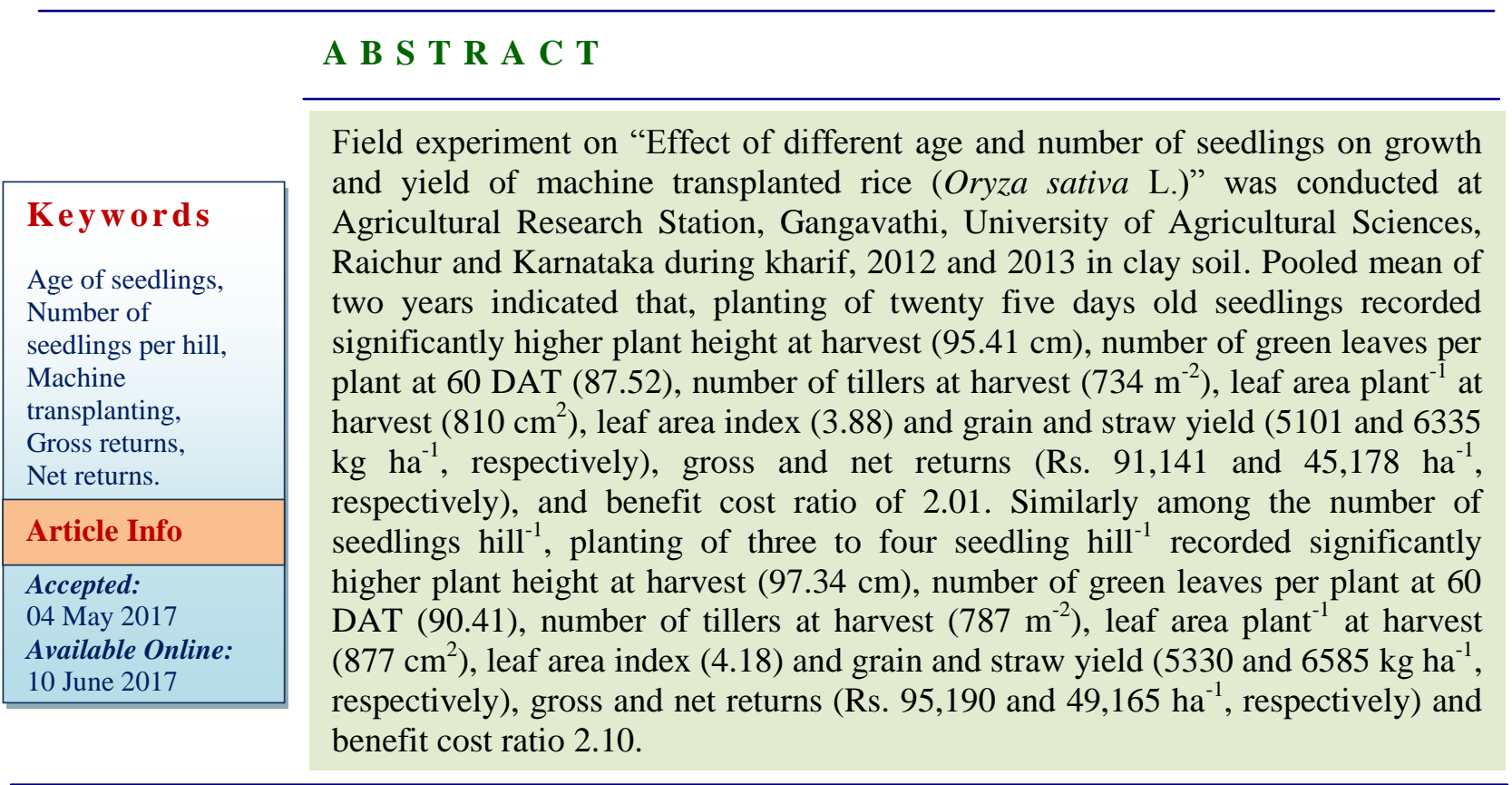

\section{Introduction}

Rice (Oryza sativa L.) is considered as the "global grain". It is the major staple food for more than half of the global population. In rice production, India ranks second as it is grown in almost all the states of the country.

Total estimated area under rice in India is 44.40 million hectares with a production of 104.32 million tonnes. West Bengal has the highest rice production, while Punjab has the highest productivity of rice among the different rice growing states of India.
Proper age of seedlings for transplanting varies with management practices, growth period, variety and growing season. In case of high yielding varieties, the seedlings should be transplanted at 4-5 leaf stage (Shastry, 1977). Twenty days old seedlings were most suitable for transplanters namely QUAT, CRRI and Yanji (Aswini et al., 2009). There is a necessary to optimize the age of seedlings for minimizing root damage for proper functioning of the transplanters (Aswini et al., 2009). 
Number of seedlings transplanted per hill varies from country to country. While in Burma, one to four seedlings are transplanted per hill, in Sri Lanka only one seedling is used. Usually, 5 to 7 seedlings are transplanted in Philippines. Results in India indicated that the number of fertile tillers were greater with 3-4 seedlings (Hedayetullaha, 1977).

Transplanting is done manually, which is tough and involves enormous drudgery and human stress in sweltering weather. It requires about 300-350 man hours per hectare, which is approximately 25 per cent of total labour requirement for paddy cultivation. Non availability of labour has compounded the situation and paddy transplanting has emerged as the problem in the major rice growing areas of this region. This results in delay in transplanting and decrease in yield. In spite of the huge labour requirement, plant to plant and row to row spacing are not achieved as the workers transplant seedlings at far wider spacing that too randomly than recommended and hence mechanical weeding is also not possible. So also, the scarcity of labour at peak demand period results an increased cost of operation and delays the transplanting operation. Hence, transplanting of paddy seedling with a suitable mechanical transplanter seems to be most appropriate and promising avenue, as it minimizes drudgery and saves much of the labour requirement (Vasudevan et al., 2014).

\section{Materials and Methods}

Under Northern dry zone of Karnataka, between 15o 15' 40" North latitude and 76o 31' 40" East longitude Agricultural Research Station, Gangavathi is situated at an altitude of $419 \mathrm{~m}$ above mean sea level where rice is the predominant irrigated crop under Tungabhadra command area which falls under the jurisdiction of University of Agricultural
Sciences, Raichur, Karnataka. Field experiment on effect of age and number of seedlings on growth and yield of machine transplanted rice was conducted during kharif, 2012 and 2013. The design used was laid in strip-plot design and soil type was medium deep black clay. The initial soil analysis of indicated electrical conductivity (2.1), soil reaction (8.2) estimated with the as outlined by Jackson (1973), available N (247.2 $\mathrm{kg} \mathrm{ha}^{-1}$ ) Subbaiah and Asija (1956), available $\mathrm{P}_{2} \mathrm{O}_{5}$ (50.2 kg ha ${ }^{-1}$ ) Olsen et al., (1954) and available $\mathrm{K}_{2} \mathrm{O}$ (357.6 $\mathrm{kg} \mathrm{ha}^{-1}$ ) Jackson (1973) at top surface $0-20 \mathrm{~cm}$ soil depth.

The experiment consisted three different age of seedlings viz., A1: twenty days old seedlings, A2: twenty five days old seedlings and A3: thirty days old seedlings and three different number of seedlings per hill planted by transplanter viz., N1: 3-4 seedlings per hill, N2: 5-6 seedlings per hill and N3:7-8 seedlings per hill. The land preparation consisted of passing of cultivator twice followed by puddling with disc puddler twice and finally levelled with spike tooth harrow and three different aged seedlings raised were transplanted on the same day.

As a pre emergent herbicide butachlor $50 \mathrm{EC}$ at the rate of 2.5 liter $\mathrm{ha}^{-1}$ was sand mixed and broadcasted over the field uniformly having thin film of water and two hand weedings taken up at 20 and 40 days after transplanting. Upto 10 days from the planting $2.5 \mathrm{~cm}$ depth of standing water was maintained and was increased to $5 \mathrm{~cm}$ until 10 days before the harvest of the crop. A fertilizer dose of (150:75:75 and $20 \mathrm{~kg} \mathrm{~N}: \mathrm{P}_{2} \mathrm{O}_{5}: \mathrm{K}_{2} \mathrm{O}$ and $\mathrm{ZnSO}_{4} / \mathrm{ha}$ ) was applied using Urea, Diammonium phosphate (DAP), Muriate of potash (MOP) and zinc sulphate, other plant protection measures were taken as and when required. At physiological maturity, the crop was harvested, dried, threshed and cleaned manually in both the years and dry weights of 
both grain and straw were recorded upon sun drying for a week. Different variable costs of items like seeds, fertilizers, irrigation, plant protection chemicals, hiring charges of transplanter, fuel cost and labour charges prevailed in market during 2012 and 2013 were considered for calculating cost of cultivation.

\section{Results and Discussion}

\section{Growth parameters}

\section{Age of seedlings}

Significant response was noticed by rice crop due to planting of different aged seedlings. Pooled data indicated that significantly higher plant height $(4.51 \%)$, number of green leaves per plant $(3.34 \%)$, number of tillers $(10.70$ $\%)$, leaf area (17.05\%), leaf area index (16.97 $\%)$ and total dry matter production at harvest (8.71) were observed by planting of twenty five days old seedlings (Tables 1 and 2) over planting of twenty days old seedlings, but was found to be on par with planting of thirty days old seedlings.

The increased height of plants might be attributed to early establishment of twenty five days old plants and subsequently more absorption of nutrients from the soil as compared to other two ages of seedlings. These results are similar with the findings of Faruk et al., (2009).

Singh and Husain (1983) also reported increased rice growth parameters with planting of 25-30 days old seedlings and mentioned that younger seedlings had lower effective tiller rate and prone to mechanical damage when compared with higher aged seedlings. Vijayalaxmi et al., (2016) reported 25 days old seedlings as the optimum age as for higher DMP with highest partitioning towards panicle followed by stem and leaf.

\section{Number of seedling per hill}

Planting of different number of seedlings per hill produced significant influence on rice growth parameters. Planting of 3-4 seedlings per hill recorded significantly higher plant height $(8.99 \%)$, number of green leaves per plant $(10.91 \%)$, number of tillers $(27.55 \%)$ (Table 1), leaf area (47.15\%), leaf are index $(47.18 \%)$ and total dry matter production at harvest $(24.71 \%)$ (Tables 1 and 2) over planting of 7-8 seedlings per hill. Planting of 5-6 seedlings per hill was the next best treatment. These findings can be corroborated with the reports of Maiti and Bhattacharya (2011) and Rasool et al., (2013) who reported that planting of fewer numbers of seedlings hill $^{-1}$ enabled the plant to produce healthy leaves and tillers which had undergone normal physiological growth and field duration, resulting in more healthy leaf area and panicles with more filled spikelets. Whereas, transplanting of 4 to 5 seedlings hill $^{-1}$ resulted in production of weak panicles with less filled spikelets. Optimum plant density for higher DMP and grain yield would be 5 seedlings hill $^{-1}$ which produced higher DM with highest partitioning towards panicle followed by stem and leaf (Vijayalaxmi et al., 2016).

\section{Yield}

\section{Age of seedlings}

Age of seedlings had significant influence on yield and significantly higher grain yield $(6.20 \%)$ and straw yield $(5.65 \%)$ was also noticed by planting of twenty five days old seedlings over planting of twenty days old seedlings (4803 $\mathrm{kg} \mathrm{ha}^{-1}$ ) and straw yield (5996 $\mathrm{kg} \mathrm{ha}{ }^{-1}$ ) (Table 3). This treatment was followed by planting of thirty days old seedlings. Kim et al., (1999) reported that young seedlings had lower effective tiller production rate when compared with more 
aged seedlings. Maximum non-bearing tillers were recorded from two weeks old seedlings and the lowest one was in four weeks old seedling and mentioned that number of unbearing tillers hill ${ }^{-1}$ increased by decreasing seedling age. This might be due to the production of secondary and tertiary tillers in the main field by low aged tillers which are incapable for production of panicle. Increased grain and straw yield due to planting of four weeks old seedlings was also reported by Bozorgi et al., (2011). Singh and Husain (1983) also reported increased rice grain yield with planting of 25-30 days old seedlings.

Table.1 Plant height, number of green leaves and number of tillers of machine transplanted rice as influenced by age and number of seedlings

\begin{tabular}{|c|c|c|c|c|c|c|c|c|c|}
\hline \multirow{2}{*}{ Treatments } & \multicolumn{3}{|c|}{$\begin{array}{c}\text { Plant height }(\mathbf{c m}) \\
\text { at harvest }\end{array}$} & \multicolumn{3}{|c|}{$\begin{array}{c}\text { Number of green } \\
\text { leaves plant }^{-1} \text { at } 60 \\
\text { DAT }\end{array}$} & \multicolumn{3}{|c|}{$\begin{array}{c}\text { Number of tillers } m^{-2} \text { at } \\
\text { harvest }\end{array}$} \\
\hline & 2012 & 2013 & $\begin{array}{c}\text { Poole } \\
\text { d }\end{array}$ & 2012 & 2013 & $\begin{array}{c}\text { Poole } \\
\text { d }\end{array}$ & 2012 & 2013 & $\begin{array}{c}\text { Poole } \\
\text { d }\end{array}$ \\
\hline \multicolumn{10}{|c|}{ Main treatments (A) } \\
\hline $\mathrm{A}_{1}$ & 89.44 & 93.13 & 91.29 & 83.00 & 86.37 & 84.69 & 657 & 668 & 663 \\
\hline $\mathrm{A}_{2}$ & 93.64 & 97.18 & 95.41 & 85.44 & 89.59 & 87.52 & 732 & 736 & 734 \\
\hline $\mathrm{A}_{3}$ & 91.71 & 94.76 & 93.24 & 84.44 & 88.37 & 86.41 & 705 & 717 & 712 \\
\hline S.Em. \pm & 0.64 & 0.73 & 0.65 & 0.41 & 0.41 & 0.50 & 9.62 & 9.22 & 9.53 \\
\hline $\begin{array}{c}\text { C.D. } \\
(\mathbf{P}=\mathbf{0 . 0 5})\end{array}$ & 2.51 & 2.87 & 2.56 & 1.59 & 1.62 & 1.98 & 37.77 & 36.20 & 37.42 \\
\hline \multicolumn{10}{|c|}{ Sub treatments $(\mathbf{N})$} \\
\hline $\mathrm{N}_{1}$ & 95.67 & 99.00 & 97.34 & 88.44 & 92.37 & 90.41 & 782 & 793 & 787 \\
\hline $\mathrm{N}_{2}$ & 91.56 & 95.02 & 93.29 & 84.56 & 88.81 & 86.69 & 698 & 708 & 703 \\
\hline $\mathrm{N}_{3}$ & 87.58 & 91.04 & 89.31 & 79.89 & 83.14 & 81.52 & 614 & 619 & 617 \\
\hline S.Em.. & 0.99 & 1.05 & 1.21 & 1.26 & 1.24 & 1.10 & 17.97 & 19.21 & 18.61 \\
\hline $\begin{array}{c}\text { C.D. } \\
(\mathbf{P}=\mathbf{0 . 0 5})\end{array}$ & 3.90 & 4.12 & 4.75 & 4.96 & 4.85 & 4.32 & 70.57 & 75.42 & 73.09 \\
\hline \multicolumn{10}{|c|}{ Interaction (A x N) } \\
\hline $\mathrm{A}_{1} \mathrm{~N}_{1}$ & 93.67 & 97.00 & 95.34 & 86.33 & 90.37 & 88.35 & 752 & 763 & 758 \\
\hline $\mathrm{A}_{1} \mathrm{~N}_{2}$ & 98.20 & $\begin{array}{c}101.8 \\
7\end{array}$ & $\begin{array}{c}100.0 \\
4\end{array}$ & 89.67 & 93.70 & 91.69 & 811 & 818 & 815 \\
\hline $\mathrm{A}_{1} \mathrm{~N}_{3}$ & 95.13 & 98.13 & 96.63 & 89.33 & 93.03 & 91.18 & 782 & 800 & 791 \\
\hline $\mathrm{A}_{2} \mathrm{~N}_{1}$ & 90.00 & 93.73 & 91.87 & 84.00 & 88.03 & 86.02 & 672 & 682 & 677 \\
\hline $\mathrm{A}_{2} \mathrm{~N}_{2}$ & 93.33 & 96.67 & 95.00 & 85.67 & 90.03 & 87.85 & 731 & 732 & 732 \\
\hline $\mathrm{A}_{2} \mathrm{~N}_{3}$ & 91.33 & 94.67 & 93.00 & 84.00 & 88.37 & 86.19 & 691 & 712 & 702 \\
\hline $\mathrm{A}_{3} \mathrm{~N}_{1}$ & 84.67 & 88.67 & 86.67 & 78.67 & 80.70 & 79.69 & 547 & 560 & 554 \\
\hline $\mathrm{A}_{3} \mathrm{~N}_{2}$ & 89.40 & 93.00 & 91.20 & 81.00 & 85.03 & 83.02 & 653 & 658 & 656 \\
\hline $\mathrm{A}_{3} \mathrm{~N}_{3}$ & 88.67 & 91.47 & 90.07 & 80.00 & 83.70 & 81.85 & 643 & 640 & 642 \\
\hline S.Em. \pm & 1.39 & 0.99 & 1.24 & 0.47 & 0.69 & 1.34 & 25.53 & 21.15 & 26.28 \\
\hline $\begin{array}{c}\text { C.D. } \\
(P=0.05)\end{array}$ & NS & NS & NS & NS & NS & NS & NS & NS & NS \\
\hline
\end{tabular}


Table.2 Leaf area per plant, leaf area index and total dry matter production of machine Transplanted rice as influenced by age and number of seedlings

\begin{tabular}{|c|c|c|c|c|c|c|c|c|c|}
\hline \multirow{2}{*}{ Treatments } & \multicolumn{3}{|c|}{$\begin{array}{c}\text { Leaf area plant } \\
\text { at harvest }\end{array}$} & \multicolumn{3}{|c|}{$\begin{array}{c}\text { Leaf area index at } \\
\text { harvest }\end{array}$} & \multicolumn{3}{|c|}{$\begin{array}{l}\text { TDMP at harvest } \\
\quad\left(\text { g plant }^{-1}\right)\end{array}$} \\
\hline & 2012 & 2013 & $\begin{array}{c}\text { Poole } \\
\text { d }\end{array}$ & 2012 & 2013 & $\begin{array}{c}\text { Poole } \\
\text { d }\end{array}$ & 2012 & 2013 & $\begin{array}{c}\text { Poole } \\
\text { d }\end{array}$ \\
\hline \multicolumn{10}{|c|}{ Main treatments (A) } \\
\hline $\mathrm{A}_{1}$ & 680 & 703 & 692 & 3.24 & 3.35 & 3.30 & 70.65 & 76.71 & 73.68 \\
\hline $\mathrm{A}_{2}$ & 805 & 814 & 810 & 3.83 & 3.88 & 3.86 & 76.93 & 83.27 & 80.10 \\
\hline $\mathrm{A}_{3}$ & 745 & 760 & 753 & 3.55 & 3.62 & 3.59 & 73.86 & 79.19 & 76.53 \\
\hline S.Em. \pm & 17.40 & 15.80 & 17.69 & 0.08 & 0.08 & 0.08 & 0.84 & 0.85 & 0.87 \\
\hline $\begin{array}{c}\text { C.D. } \\
(P=0.05)\end{array}$ & 68.34 & 62.04 & 67.47 & 0.32 & 0.30 & 0.33 & 3.29 & 3.34 & 3.43 \\
\hline \multicolumn{10}{|c|}{ Sub treatments $(\mathrm{N})$} \\
\hline $\mathrm{N}_{1}$ & 873 & 880 & 877 & 4.16 & 4.19 & 4.18 & 82.02 & 89.08 & 85.55 \\
\hline $\mathrm{N}_{2}$ & 768 & 795 & 782 & 3.66 & 3.79 & 3.73 & 73.11 & 79.20 & 76.16 \\
\hline $\mathrm{N}_{3}$ & 589 & 603 & 596 & 2.80 & 2.87 & 2.84 & 66.31 & 70.89 & 68.60 \\
\hline S.Em. \pm & 25.69 & 25.89 & 26.32 & 0.12 & 0.12 & 0.13 & 1.54 & 1.71 & 1.77 \\
\hline $\begin{array}{c}\text { C.D. } \\
(P=0.05)\end{array}$ & $\begin{array}{c}100.8 \\
8\end{array}$ & 101.64 & 103.33 & 0.48 & 0.49 & 0.49 & 6.06 & 6.70 & 6.96 \\
\hline \multicolumn{10}{|c|}{ Interaction $(\mathbf{A} \times \mathbf{N})$} \\
\hline $\mathrm{A}_{1} \mathrm{~N}_{1}$ & 839 & 852 & 846 & 4.00 & 4.05 & 4.03 & 78.10 & 85.27 & 81.69 \\
\hline $\mathrm{A}_{1} \mathrm{~N}_{2}$ & 907 & 920 & 914 & 4.32 & 4.38 & 4.35 & 85.87 & 93.78 & 89.83 \\
\hline $\mathrm{A}_{1} \mathrm{~N}_{3}$ & 873 & 866 & 870 & 4.15 & 4.13 & 4.14 & 82.09 & 88.20 & 85.15 \\
\hline $\mathrm{A}_{2} \mathrm{~N}_{1}$ & 734 & 774 & 754 & 3.50 & 3.69 & 3.60 & 70.40 & 76.22 & 73.31 \\
\hline $\mathrm{A}_{2} \mathrm{~N}_{2}$ & 810 & 824 & 818 & 3.86 & 3.93 & 3.90 & 75.23 & 82.62 & 78.93 \\
\hline $\mathrm{A}_{2} \mathrm{~N}_{3}$ & 761 & 786 & 774 & 3.62 & 3.74 & 3.68 & 73.69 & 78.77 & 76.23 \\
\hline $\mathrm{A}_{3} \mathrm{~N}_{1}$ & 468 & 483 & 476 & 2.23 & 2.30 & 2.27 & 63.44 & 68.65 & 66.05 \\
\hline $\mathrm{A}_{3} \mathrm{~N}_{2}$ & 698 & 698 & 698 & 3.32 & 3.32 & 3.32 & 69.69 & 73.42 & 71.56 \\
\hline $\mathrm{A}_{3} \mathrm{~N}_{3}$ & 601 & 628 & 615 & 2.86 & 2.99 & 2.93 & 65.79 & 70.60 & 68.20 \\
\hline S.Em. \pm & 33.61 & 35.23 & 29.07 & 0.16 & 0.17 & 0.14 & 2.33 & 1.41 & 1.74 \\
\hline $\begin{array}{c}\text { C.D. } \\
(P=0.05)\end{array}$ & NS & NS & NS & NS & NS & NS & NS & NS & NS \\
\hline
\end{tabular}


Table.3 Grain yield, straw yield and harvest index of machine transplanted rice as influenced by age and number of seedlings

\begin{tabular}{|c|c|c|c|c|c|c|c|c|c|}
\hline \multirow[b]{2}{*}{ Treatments } & \multicolumn{3}{|c|}{ Grain yield (kg/ha) } & \multicolumn{3}{|c|}{ Straw yield (kg/ha) } & \multicolumn{3}{|c|}{ Harvest index } \\
\hline & 2012 & 2013 & $\begin{array}{c}\text { Poole } \\
\text { d }\end{array}$ & 2012 & 2013 & $\begin{array}{c}\text { Poole } \\
\text { d }\end{array}$ & 2012 & 2013 & $\begin{array}{c}\text { Poole } \\
\text { d }\end{array}$ \\
\hline \multicolumn{10}{|c|}{ Main treatments (A) } \\
\hline A1 & 4753 & 4853 & 4803 & 5930 & 6061 & 5996 & 0.45 & 0.45 & 0.45 \\
\hline $\mathrm{A} 2$ & 5080 & 5122 & 5101 & 6245 & 6425 & 6335 & 0.45 & 0.44 & 0.45 \\
\hline A3 & 4841 & 4936 & 4889 & 6049 & 6195 & 6122 & 0.44 & 0.45 & 0.45 \\
\hline S.Em. \pm & 61 & 52 & 57 & 60 & 66 & 63 & 0.00 & 0.00 & 0.00 \\
\hline $\begin{array}{c}\text { C.D. } \\
(\mathrm{P}=0.05)\end{array}$ & 241 & 202 & 226 & 234 & 261 & 247 & NS & NS & NS \\
\hline \multicolumn{10}{|c|}{ Sub treatments $(\mathbf{N})$} \\
\hline $\mathrm{N}_{1}$ & 5295 & 5365 & 5330 & 6515 & 6655 & 6585 & 0.45 & 0.45 & 0.45 \\
\hline $\mathrm{N}_{2}$ & 4834 & 4903 & 4869 & 6048 & 6208 & 6128 & 0.44 & 0.44 & 0.44 \\
\hline $\mathrm{N}_{3}$ & 4546 & 4643 & 4595 & 5661 & 5818 & 5740 & 0.45 & 0.45 & 0.45 \\
\hline S.Em. \pm & 67 & 67 & 67 & 86 & 85 & 85 & 0.00 & 0.00 & 0.00 \\
\hline $\begin{array}{c}\text { C.D. } \\
(P=0.05)\end{array}$ & 263 & 262 & 263 & 337 & 334 & 332 & NS & NS & NS \\
\hline \multicolumn{10}{|c|}{ Interaction $(\mathrm{A} \times \mathrm{x})$} \\
\hline $\mathrm{A}_{1} \mathrm{~N}_{1}$ & 5067 & 5166 & 5117 & 6368 & 6513 & 6441 & 0.44 & 0.44 & 0.44 \\
\hline $\mathrm{A}_{1} \mathrm{~N}_{2}$ & 5597 & 5635 & 5616 & 6692 & 6853 & 6773 & 0.46 & 0.45 & 0.46 \\
\hline $\mathrm{A}_{1} \mathrm{~N}_{3}$ & 5220 & 5295 & 5258 & 6485 & 6599 & 6542 & 0.45 & 0.45 & 0.45 \\
\hline $\mathrm{A}_{2} \mathrm{~N}_{1}$ & 4715 & 4819 & 4767 & 5953 & 6023 & 5988 & 0.44 & 0.45 & 0.45 \\
\hline $\mathrm{A}_{2} \mathrm{~N}_{2}$ & 4972 & 4970 & 4971 & 6138 & 6390 & 6264 & 0.45 & 0.44 & 0.45 \\
\hline $\mathrm{A}_{2} \mathrm{~N}_{3}$ & 4816 & 4920 & 4868 & 6052 & 6210 & 6131 & 0.44 & 0.44 & 0.44 \\
\hline $\mathrm{A}_{3} \mathrm{~N}_{1}$ & 4478 & 4574 & 4526 & 5468 & 5647 & 5558 & 0.45 & 0.45 & 0.45 \\
\hline $\mathrm{A}_{3} \mathrm{~N}_{2}$ & 4671 & 4762 & 4717 & 5904 & 6032 & 5968 & 0.44 & 0.44 & 0.44 \\
\hline $\mathrm{A}_{3} \mathrm{~N}_{3}$ & 4488 & 4594 & 4541 & 5610 & 5775 & 5693 & 0.44 & 0.45 & 0.45 \\
\hline S.Em. \pm & 106 & 103 & 100 & 188 & 116 & 142 & 0.01 & 0.01 & 0.01 \\
\hline $\begin{array}{c}\text { C.D. } \\
(P=0.05)\end{array}$ & NS & NS & NS & NS & NS & NS & NS & NS & NS \\
\hline
\end{tabular}


Table.4 Gross returns, net returns and benefit cost ratio of machine transplanted rice as influenced by age and number of seedlings

\begin{tabular}{|c|c|c|c|c|c|c|c|c|c|}
\hline \multirow{2}{*}{ Treatments } & \multicolumn{3}{|c|}{ Gross returns $\left(\mathrm{ha}^{-1}\right)$} & \multicolumn{3}{|c|}{ Net returns $\left(\mathrm{ha}^{-1}\right)$} & \multicolumn{3}{|c|}{$\mathbf{B}: \mathbf{C}$} \\
\hline & 2012 & 2013 & Pooled & 2012 & 2013 & Pooled & 2012 & 2013 & Pooled \\
\hline \multicolumn{10}{|c|}{ Main treatments $(\mathrm{A})$} \\
\hline A1 & 89116 & 82499 & 85808 & 47331 & 33283 & 40307 & 2.13 & 1.68 & 1.91 \\
\hline A2 & 95187 & 87094 & 91141 & 52921 & 37435 & 45178 & 2.26 & 1.76 & 2.01 \\
\hline A3 & 90773 & 83939 & 87356 & 48537 & 34205 & 41371 & 2.15 & 1.69 & 1.92 \\
\hline S.Em. \pm & 702 & 702 & 702 & 716 & 716 & 716 & 0.02 & 0.01 & 0.01 \\
\hline $\begin{array}{c}\text { C.D. } \\
(\mathrm{P}=0.05)\end{array}$ & 2756 & 2756 & 2756 & 2812 & 2812 & 2812 & 0.09 & 0.04 & 0.04 \\
\hline \multicolumn{10}{|c|}{ Sub treatments $(\mathbf{N})$} \\
\hline N1 & 99211 & 91168 & 95190 & 56891 & 41439 & 49165 & 2.35 & 1.84 & 2.10 \\
\hline $\mathrm{N} 2$ & 90645 & 83415 & 87030 & 48580 & 33907 & 41244 & 2.16 & 1.69 & 1.93 \\
\hline N3 & 85220 & 78950 & 82085 & 43317 & 29576 & 36447 & 2.03 & 1.60 & 1.82 \\
\hline S.Em. \pm & 946 & 946 & 946 & 977 & 977 & 977 & 0.03 & 0.03 & 0.02 \\
\hline $\begin{array}{c}\text { C.D. } \\
(\mathrm{P}=0.05)\end{array}$ & 3715 & 3715 & 3715 & 3835 & 3835 & 3835 & 0.12 & 0.012 & 0.06 \\
\hline \multicolumn{10}{|c|}{ Interaction (A x N) } \\
\hline A1N1 & 95021 & 87867 & 91444 & 53059 & 38489 & 45774 & 2.26 & 1.78 & 2.02 \\
\hline $\mathrm{A} 1 \mathrm{~N} 2$ & 104761 & 95637 & 100199 & 62212 & 45743 & 53977 & 2.47 & 1.92 & 2.20 \\
\hline A1N3 & 97851 & 89999 & 93925 & 55403 & 40084 & 47744 & 2.30 & 1.81 & 2.06 \\
\hline A2N1 & 88442 & 81928 & 85185 & 46677 & 32729 & 39703 & 2.12 & 1.67 & 1.90 \\
\hline $\mathrm{A} 2 \mathrm{~N} 2$ & 93179 & 84627 & 88903 & 50971 & 35031 & 43001 & 2.21 & 1.71 & 1.96 \\
\hline A2N3 & 90313 & 83688 & 87000 & 48092 & 33962 & 41026 & 2.14 & 1.69 & 1.92 \\
\hline A3N1 & 83885 & 77702 & 80793 & 42256 & 28629 & 35442 & 2.01 & 1.58 & 1.80 \\
\hline A3N2 & 87620 & 81018 & 84319 & 45580 & 31530 & 38555 & 2.09 & 1.64 & 1.87 \\
\hline A3 N3 & 84156 & 78130 & 81143 & 42115 & 28570 & 35342 & 2.00 & 1.58 & 1.79 \\
\hline S.Em. \pm & 1599 & 1599 & 1599 & 1774 & 1774 & 1774 & 0.06 & 0.08 & 0.05 \\
\hline $\begin{array}{c}\text { C.D. } \\
(\mathrm{P}=0.05)\end{array}$ & NS & NS & NS & NS & NS & NS & NS & NS & NS \\
\hline
\end{tabular}


Fig.1 Grain and Straw yield of machine transplanted rice as influenced by Age and number of seedlings

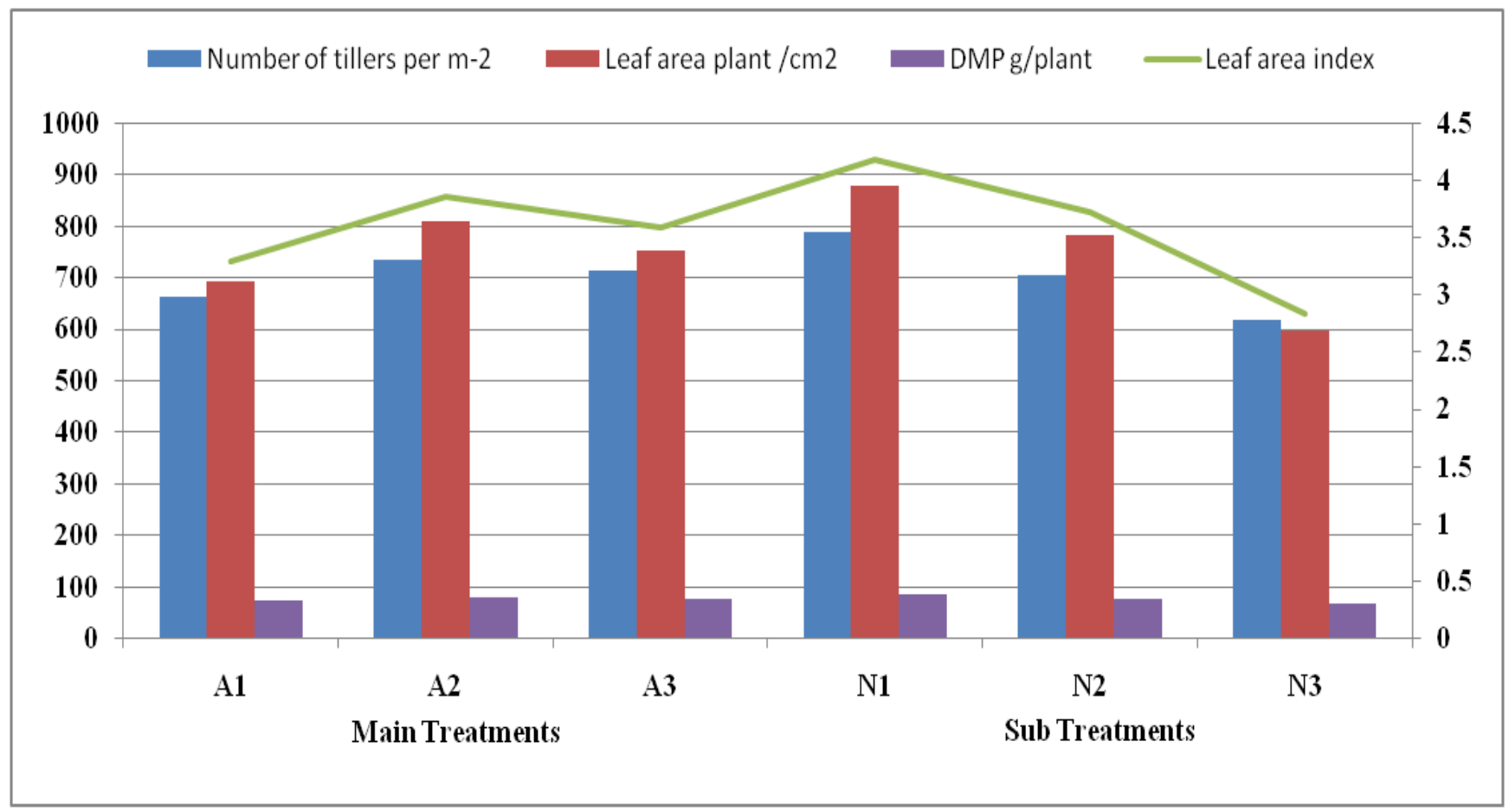

Fig.2 Economics of machine transplanted rice as influenced by age and number of seedlings

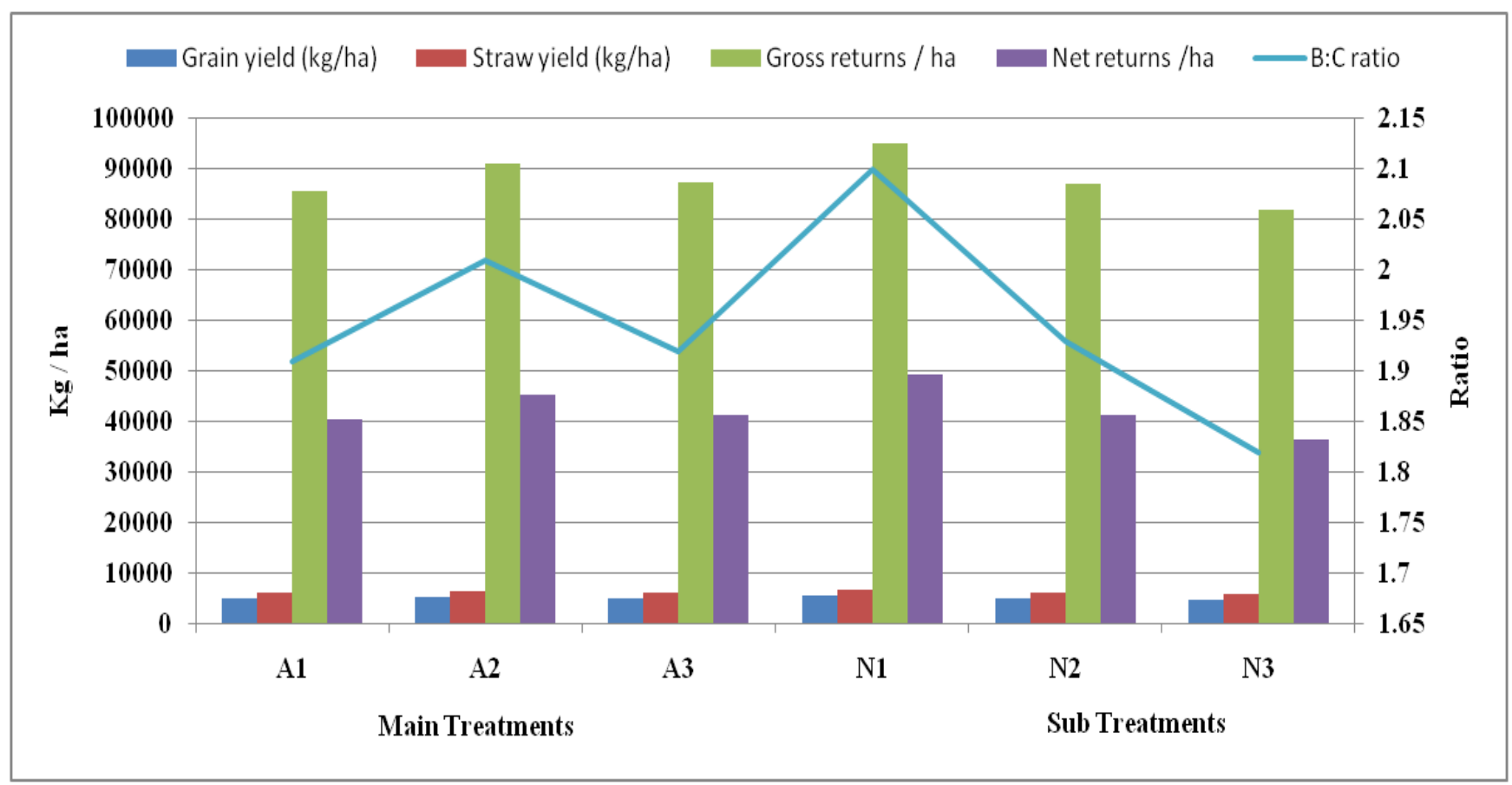




\section{Number of seedling per hill}

Planting of different number of seedlings per hill noticed significant influence on yield and yield parameters. Planting of 3-4 seedlings per hill produced significantly higher grain yield $(16.00 \%)$ and straw $(14.72 \%)$ as compared to planting of 7-8 seedlings per hill $\left(4595 \mathrm{~kg} \mathrm{ha}^{-1}\right)$ and straw yield (5740 $\left.\mathrm{kg} \mathrm{ha}^{-1}\right)$ (Table 3). However, it was followed by planting of 5-6 seedlings per hill $\left(4868 \mathrm{~kg} \mathrm{ha}^{-1}\right)$ and straw yield $\left(6128 \mathrm{~kg} \mathrm{ha}^{-1}\right)$. This may be due to healthy and efficient individual plant growth at lesser seedling density. The higher yield with low seedling density might be due to higher percentage of productive total tillers and more interception of light. Also, grain filling which is the process of remobilization from stored reserves, particularly from stem, leaves, and from current photosynthesis. So, it may be inferred that the effectiveness of grain filling is decided by the conditions of particular tiller. Hence, planting of fewer seedlings resulted in higher grain yield (Rasool et al., 2013). Such increase in yield contributing parameters with fewer number of seedlings per hill were also reported by Bozorgi et al., (2011) and Maiti and Bhattacharya (2011).

\section{Economics}

\section{Age of seedlings}

Planting of twenty five days old seedlings recorded significantly higher gross returns (Rs. 91,141), net returns (Rs. 45,178) and B: $\mathrm{C}$ (2.01) as compared to planting of twenty days old seedlings which recorded gross returns (Rs. 85,808), net returns (Rs. 40,307) and B: C (1.91) and it was followed by planting of thirty days old seedlings (Table 4). The increased net returns in case of planting of twenty five days old seedlings was obviously due to the higher grain and straw yield.

\section{Number of seedling per hill}

Planting of 3-4 seedlings per hill recorded significantly higher gross returns (Rs. 95,190), net returns (Rs. 49,165) and B: C (2.10) as compared planting of 7-8 seedlings per hill gross returns (Rs. 82,085), net returns (Rs. 36,447) and B: C (1.82) (Table 4) and this may be attributed to the higher grain and straw yield obtained in this particular treatment compared to other planting geometry under study.

From the investigation it may be concluded that, planting of twenty five days old seedlings was found to be the best seedling age over 20 and 30 days for transplanting by self-propelled mechanical transplanter. Planting of 3-4 seedlings per hill was optimum for transplanting with self-propelled mechanical transplanter when compared to 56 and 7-8 seedlings per hill.

\section{References}

Anonymous, 2011, Ministry of Agriculture, Govt. of India. http:// www.indiastat.com.

Aswini GK, Swain S and Debaraj B 2009. Effect of seedling age on performance of rice transplanter. Agricultural Mechanization in Asia, Africa and Latin America, 40(3): 41-46.

Behera BK 2000. Investigation on puddle soil characteristics in relation to performance of self-propelled rice transplanter. Un-published Ph.D. Thesis G. B. Pant University of Agriculture and Technology, Pantnagar, UP.

Bozorgi HR, Amin Faji, Khasravi Danesh, Abdolkarim K, Ebrahim A and Freshieh T 2011. Effect of plant density on yield and yield component of rice. World Applied Sci. J., 12 (11):2053-2057.

Faruk MO, Rahman MA and Hassan MA 2009. Effect of seedling age and 
number of seedling per hill on the yield and yield contributing characters of Dhan 33. Int. J. Sustain. Crop Prod., 4(1):58-61.

Hedayetullaha S 1977. A study on the effect of cultural factor in transplanted paddy on the behaviour of some plant characteristics influencing yield. Indian J. Agric. Sci., 17: 39-80.

Jackson M L 1973. Soil Chemical Analysis, (Ed.). Prentice-Hall of India, Pvt. Ltd., New Delhi, pp. 121-125.

Kim SS, Kim BK, Choi MG, Back MH, Choi WY and Lee SY 1999. Effect of seedling age on

Growth and yield of machine transplanted rice in southern plain region. Korean $\mathrm{J}$.

Crop Sci. 44(2): 122-128

Maiti PK and Bhattacharya B 2011. Effect of seedling rate and number of seedlings per hill on the growth and yield of hybrid rice (Oryza sativa L.) grown in dry (boro) season. Crop Res., 42 (1, 2 \& 3): 18-22.

Naidu GJ, Rao KT, Rao AU and Reddy DS 2013. Age of seedling and planting pattern on grain yield, protein content, NPK uptake and post-harvest nutrient status of rice under SRI. J. Acad. Indust. Res., 2 (6): 334-337.

Olsen SR, Cole CL, Watanabe FS and Deaw DA 1954. Estimation of available phosphorous in soils by extraction with sodium bicarbonate. United States Departments of Agriculture circular, p.939.

Rasool F, Habib R and Bhat MI 2013. Agronomic evaluation of rice (Oryza sativa L.) for plant spacing and seedlings per hill under temperate conditions. Pak. J. Agric. Sci. 9(2): 169172.

Shastry SVS 1977. Research note. AICRP on rice research project. Int. Rice Com. Newslet., 26(2): 11.

Singh G and Husain UK 1983. Modification and testing of a manual rice transplanter. AMA, 14(2): 25-30.

Subbaiah BV and Asija GL 1956. Rapid procedure for determination of available nitrogen in soils. Current. Sci., 31: 196.

Vasudevan SN, Basangouda, Mathad RC, Doddagoudar SR and Shakuntala NM 2014. Standardization of Seedling Characteristics for Paddy Transplanter. Journal of Advanced Agricultural Technologies 1(2), 141-146.

Vijayalaxmi, G., Sreenivas, G., Leela Rani, P. and Ram Prakash, T., 2016. Influence of Plant Densities and Age of Seedlings on Dry Matter Partitioning and Grain Yield Potential of Transplanted Rice (Oryza sativa L.). International Journal of Current Research in Biosciences and Plant Biology, 3(4): 51-55.

\section{How to cite this article:}

Negalur, R.B. and Halepyati, A.S. 2017. Growth and Yield of Machine Transplanted Rice (Oryza sativa L.) as Influenced by Age and Number of Seedlings. Int.J.Curr.Microbiol.App.Sci. 6(6): 376-385. doi: https://doi.org/10.20546/ijcmas.2017.606.044 